•研究报告・

\title{
人为干扰对溪流鱼类功能多样性及其纵向梯度格 局的影响
}

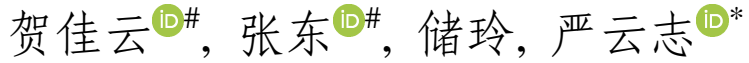

安徽师范大学生态与环境学院, 省部共建皖江流域退化生态系统修复与重建协同创新中心, 安徽芜湖 241000

摘要: 溪流鱼类多样性沿着河流纵向梯度的空间分布规律已得到大量报道, 但这些研究大多聚焦基于物种组成的分类 $\alpha$ 多样 性，而有关分类 $\beta$ 多样性和功能多样性的纵向梯度分布规律及其对人类干扰的响应研究较少。本文以青七江上游 3 条人为干扰 程度不同的河源溪流为研究区域, 比较研究了人为干扰对溪流鱼类功能 $\alpha$ 和 $\beta$ 多样性及其纵向梯度分布格局的影响。结果显示, 人类干扰改变了河源溪流鱼类功能多样性的纵向梯度格局——由线性变化变为二项式分布。此外, 我们发现, 人为干扰导致 土著种被本地入侵种取代, 且较强的土地利用和水污染排放可能增大环境的不连续性, 而群落周转和嵌套变化往往取决于环 境的变化。尽管功能 $\beta$ 多样性由嵌套成分主导, 但周转成分占比相对于人为干扰较小的溪流而言明显增加。人为干扰显著改 变了受干扰溪流鱼类的物种组成和功能多样性, 且功能多样性的纵向梯度格局在不同的多样性指标上存在差异。本研究强调, 在评估人为干扰下多样性的变化时, 需要从多方面考虑, 包括空间尺度和多样性指标等。

关键词: 河流连续体; 功能多样性; 物种周转; 物种嵌套; 人为干扰

贺佳云, 张东, 储玲, 严云志 (2021) 人为干扰对溪流鱼类功能多样性及其纵向梯度格局的影响. 生物多样性, 29, 927-937. doi: 10.17520/biods. 2020434.

He JY, Zhang D, Chu L, Yan YZ (2021) Anthropogenic disturbances affect the functional diversity of stream fishes and its longitudinal patterns in China. Biodiversity Science, 29, 927-937. doi: 10.17520/biods.2020434.

\section{Anthropogenic disturbances affect the functional diversity of stream fishes and its longitudinal patterns in China}

\author{
Jiayun He ${ }^{\mathbb{D} \#}$, Dong Zhang ${ }^{\mathbb{1} \#}$, Ling Chu, Yunzhi Yan ${ }^{(\mathbb{D} *}$
}

School of Ecology and Environment, Anhui Normal University, and Collaborative Innocation Center of Reconstruction of Degraded Ecosystem in Wanjiang Basin Co-founded by Anhui Province and Ministry of Education, Wuhu, Anhui 241000

\begin{abstract}
Aims: The distribution and abundance of stream fishes are influenced by historical events as well as abiotic and biotic factors. Due to the high spatial heterogeneity and temporal differentiation of stream environmental characteristics, the composition and abundance of stream fish communities vary on spatial and temporal scales. On this basis, longitudinal patterns in the diversity and distribution of stream fish along upstream-downstream gradients have garnered the widespread attention of ecologists. Previous studies relevant to the longitudinal patterns in stream fish diversity along the upstream-downstream gradient mostly focused on taxonomic $\alpha$ diversity, while taxonomic $\beta$ diversity and functional diversity have been given less attention. Therefore, this study aimed to (1) determine the effects of anthropogenic disturbances on species composition of fishes by comparing diversity across three streams, and determine the key species contributing to the between-stream variation in fish assemblages; (2) examine the influences of anthropogenic disturbances on functional diversity, and examine if differences in functional diversity relate to $\alpha$ and $\beta$ diversity; (3) to assess whether the between-stream variations in functional diversity show dependence on the spatial positions along the river continuum or not, and further assess the effects of anthropogenic disturbances on the longitudinal patterns in the

收稿日期: 2020-11-20; 接受日期: 2021-02-09

基金项目: 国家自然科学基金(31500452; 31372227)、安徽省水产产业技术体系项目(皖农科（2016）84号)和重要生物资源保护与利用研究安徽省省级 重点实验室经费

\# 共同第一作者 Co-first authors

* 通讯作者 Author for correspondence. E-mail: yanyunzhi@ahnu.edu.cn
\end{abstract}


functional $\alpha$ and $\beta$ diversities of stream fishes.

Methods: In this study, we focused on the functional diversity of fishes, which is an effective measure for assessing the response of communities to environmental changes and predicting changes in ecosystem functioning. Using data collected in three headwater streams of the Qingyi River, China, that experience different extents of anthropogenic disturbances, we examined the effects of anthropogenic disturbances on the longitudinal patterns in the functional $\alpha$ and $\beta$ diversities of streams fishes. We considered multiple metrics of the $\alpha$ diversity, including the functional richness, evenness and divergence, which measure different aspects of functional space and abundance distribution. This work also analyzed $\beta$ diversity, including its turnover and nestedness components, which can provide additional insights into the processes of community changing.

Results: We found that in the less disturbed stream, functional richness increased but divergence decreased downstream, and functional $\beta$ diversity decreased downstream. On the other hand, in heavily disturbed streams functional richness was higher in the upstream-midstream but lower in the downstream, and functional divergence decreased in the upstream-midstream and $\beta$ diversity increased in the downstream. Anthropogenic disturbances significantly altered species compositions and functional diversity of fishes in the heavily disturbed streams, and the mode and degree to which functional diversity varied related to both the metrics of functional diversity and the spatial position along river continuum. As a result, anthropogenic disturbances caused a shift in the longitudinal patterns of functional diversity, from linear variation in less disturbed streams to unimodal distribution in heavily disturbed streams. Similarly, variations in the functional $\beta$ diversity of fishes mainly occurred in the lower segments, but not in the upper segments, and were derived by the turnover component. We found anthropogenic disturbances caused the endemic species to be replaced by the native-invasive species. Heavy land use and polluting emission could increase the environmental discontinuity, leading to community turnover and nestedness changing. Although functional $\beta$ diversity was dominated by the nestedness component, the proportion of the turnover component increased significantly compared with less disturbed streams.

Conclusion: Our results highlight the need for a multifaceted approach, including spatial scale and diversity metrics, in assessing the diversity changes of stream fishes under anthropogenic pressure.

Key words: river continuum; functional diversity; community turnover; community nestedness; anthropogenic disturbance

人类活动改变了溪流中的理化条件和生态过 程, 引起鱼类群落物种组成和数量的变化, 表现为 土著物种丢失和广布物种获得两个过程(Allan, 2004), 物种取代过程不仅改变了局域群落的物种 多寡(Sax \& Gaines, 2003), 也引起区域群落的物种 不相似性变化(Villéger et al, 2014), 进而对溪流鱼 类多样性构成严重威胁(张东等, 2018)。研究溪流鱼 类多样性对人类活动的响应, 是溪流生态系统及其 鱼类多样性保护和管理的科学基础, 也是淡水生态 学领域的一个重要研究课题(Dudgeon et al, 2006)。

沿着“上游-下游”的河流纵向梯度, 水文、水流 流态、能量输入等系列环境因子呈现出显著的空间 异质性(Poff \& Ward, 1990), 进而引起溪流鱼类群 落物种组成和数量特征的变化(Wang et al, 2001)。溪 流鱼类的物种丰富度从源头至河口, 其变化模式大 多为钟形, 即物种数从上游至中游逐渐上升, 在中 游或中下游河段达到最大值, 至下游相对下降(Sui et al, 2014)。人类干扰改变了鱼类局域群落的物种 丰富度, 因而也可能会引起物种丰富度纵向梯度分
布规律的变化(Li et al, 2018), 然而, 基于物种丰富 度(即分类 $\alpha$ 多样性)无法解释生物群落与环境特征 之间的关系(Zhang et al, 2020)。因此，基于特征的测 量作为一种有前景的淡水生物评价方法受到了越 来越多的关注, 如功能群多样性(张东等, 2018)和功 能多样性(Ding et al, 2017; Li et al, 2019)。尽管功能 群多样性在鱼类生态学中得到了广泛应用, 但其测 度的功能性状较少, 呈现的功能多样性实际上是功 能群的数量, 无法呈现同一功能类型不同物种间、 尤其是同一物种不同种群间的功能特征差异, 进而 导致其研究结果所反映的生态系统功能等信息不 完整(张东等, 2018)。功能多样性在评价群落对环境 变化的响应和预测生态系统功能变化方面可能更 好(Tolonen et al, 2017; Göthe et al, 2017), 同时作为 生物多样性的一个关键指标, 功能多样性有助于跨 环境梯度或空间尺度解析群落构建机制(Swenson et al, 2011; Münkemüller et al, 2012)。另外, 功能多样 性的多种度量指数可以描述局域群落功能属性的 不同方面, 如功能丰富度描述物种在群落中所占据 
的功能空间的大小, 功能均匀度衡量物种功 能性状在已占据的空间内分布是否均匀, 功能离散 度衡量群落中功能性状多度分布的最大离散程度 (Petchey \& Gaston, 2006)。

与 $\alpha$ 多样性(量化局域群落的物种多寡)不同, $\beta$ 多样性量化的是群落间的物种变化幅度(Whittaker, 1960)。群落间的物种组成变化无外乎物种丢失与物 种获得(Villéger et al, 2014)。当只有物种丢失或物种 获得时, 小群落是大群落的一个子集, 其群落变化 呈嵌套模式; 若兼有物种丢失和获得, 则表现为群 落周转格局(Leprieur et al, 2011)。可见, $\beta$ 多样性可 被分解为嵌套和周转 2 个组分(Baselga, 2010), 前者 一般发生于连续性环境变化 (Taylor \& Warren, 2001), 后者则多见于间断性环境变化(Mazzoni \& Lobón-Cerviá, 2008), 这表明 $\beta$ 多样性分解有助于认 识生物群落空间变异的生态过程(Soininen et al, 2018)。

先前有关溪流鱼类多样性的纵向梯度格局及 其对人类干扰的响应研究大多聚焦于基于物种组 成的局域多样性(物种 $\alpha$ 多样性), 对功能 $\beta$ 多样性的 纵向梯度格局及其如何响应人类干扰尚不清楚。本 研究以我国亚热带地区青七江流域上游 3 条人为干 扰强度不同的河源溪流为研究区域, 自上游至下游 连续设置研究样点进行取样; 基于采集到的渔获物, 结合鱼类功能特征, 着重比较研究人类干扰对溪流 鱼类的功能 $\alpha$ 多样性和 $\beta$ 多样性及其纵向梯度格局 的影响。具体目标包括: (1)确定人为干扰强度不同 的溪流鱼类物种组成的差异, 以及影响溪流间鱼类 群落组成差异的关键种; (2)溪流间的功能 $\alpha$ 和 $\beta$ 多样 性沿河流纵向梯度是否表现出不同的变化规律; (3) 人为干扰如何影响功能 $\alpha$ 和 $\beta$ 多样性及其纵向分布 规律。

\section{1 材料与方法}

\section{1 研究区域}

青七汇发源于黄山北麓, 呈东北流向汇入长 江。属亚热带湿润季风气候, 全年四季分明; 年均 气温 $17.8^{\circ} \mathrm{C}\left(-2.1^{\circ} \mathrm{C}-27.5^{\circ} \mathrm{C}\right)$, 年降水量约 $2,000 \mathrm{~mm}$, 主要集中在4-9月, 占全年降水量的79\%。舒溪、麻 溪和浦溪是该流域的河源溪流，受到不同程度的人 为干扰, 其中麻溪每年排放的污染物, 如化学需氧 量 $(\mathrm{COD})$ 、氨氮 $\left(\mathrm{NH}_{3}-\mathrm{N}\right)$ 和总磷 $(\mathrm{TP})$ 最高, 约为舒溪
的 4 倍, 浦溪是舒溪的 2 倍多。另外, 浦溪的城镇和 农田土地利用比例最高(超过20\%), 麻溪其次(超过 $10 \%$ ), 舒溪最低(少于5\%) (附录1)。此外，土地利用 比例沿坡度变化较大, 从高坡度至低坡度, 3 条研究 河流均呈现出上升趋势(附录2)。

\section{2 样点设置与鱼类采集}

基于栖息地的可达性和可操作性，3条研究河 流均间隔2-3 km连续设置采样点。选择的采样点均 可涉水(水深小于 $1 \mathrm{~m}$ ), 各样点采样河长为 $50 \mathrm{~m}$, 且 都包含两种栖息地斑块类型(急滩和深潭)。于 2011 年5月和10月对样点各进行 1 次鱼类采集, 舒溪、麻 溪和浦溪样点数分别是 11 个、 12 个和 9 个(图1)。采 用背式电鱼器(CWB-2000 P, China; 12 V import, 250 $\mathrm{V}$ export)直接涉水取样(经安徽省渔业渔政管理局 批准), 采样时间30 min。依据Nelson (2006)和陈小 勇(2013)的目科级分类系统，在鱼类新鲜状态下进 行鉴定(吻虾虎鱼(Rhinogobius sp.)仅鉴定至属的水 平)、计数, 疑难种保存在 $8 \%$ 的甲醛溶液中带回实验 室做进一步的鉴定。

\section{3 物种特征测量}

参照Villéger等(2008)的方法, 针对栖息地利 用、运动以及摄食等 3 方面, 测量体长、眼径、头长 等功能特征。采集到的渔获物按每种 30 尾成鱼进行

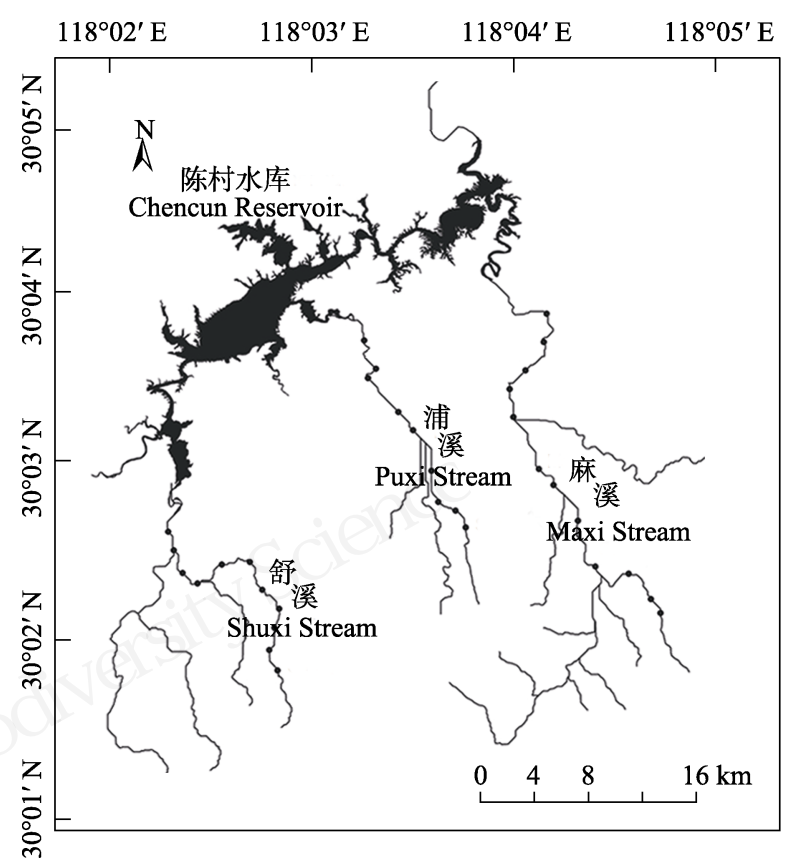

图1 青尤江舒溪、麻溪、浦溪鱼类采样点

Fig. 1 Fish samplings sites of Shuxi, Maxi and Puxi Stream, Qingyi River, Anhui 
功能特征测量, 不足30尾的物种则测量全部成鱼。 平均性状值是根据个体测量计算的, 假设每个物种 的种内变异小于种间变异(Dumay et al, 2004) (表1)。

\section{4 多样性计算}

我们选取 3 个多样性指数计算功能 $\alpha$ 多样性: 功 能丰富度(FRic)、功能离散度(FDiv)以及功能均匀度 (FEve) (Mason et al, 2005; Villéger et al, 2008) (附录 3)。利用R 3.4.1中“FD”软件包进行计算(Laliberte \& Legendre, 2010)。

参照Villéger等(2013)的方法, 根据每个群落中 所有物种的特征性状所占的空间体积以及两两样 点间的分类/功能组成, 计算功能 $\beta$ 多样性 ( $\beta s ø r)$, 并 参照Baselga (2010)的方法, 将其分解为周转 ( $\beta$ tur) 和嵌套 (Bnes) 2 个组分(附录 3 )。

\section{5 数据分析}

根据距河源距离将 3 条研究河流分成上 (0-15 km)、中(15-25 km)、下(25-40 km) 3个河段, 采用多元统计分析方法检验溪流和河段对物种组 成和功能多样性的影响。首先, 在Primer 7.0软件中, 对鱼类物种组成数据进行 $\operatorname{Lg}(1+x)$ 转换, 运用双因 素交互相似性分析(two-way crossed ANOSIM)检验 溪流和河段对群落结构空间变化的影响, 并运用非 度量多维标度法 (non-metric multidimensional scaling, nMDS)构建鱼类群落物种组成的二维双标 图，将其空间变化进行直观化。其次，运用相似百 分比分析(similarity percentages, SIMPER)确定物种 组成中对河流间的差异有贡献的关键物种。在SPSS 22.0 软件中, 使用双因素方差分析 (two-way ANOVA)分别检验溪流和河段对功能多样性各个指
数的影响。此外, 考虑到纵向梯度下鱼类功能多样 性的潜在空间格局，采用线性模型和二次项模型检 验距河源距离同功能多样性的关系。根据各模型的 相关性 $R$ 值和显著性 $P$ 值, 确定解释这种关系的最 优模型, 3条研究河流独立进行, 视 $P<0.1$ 边缘显著 差异有统计学意义(Lin et al, 2020; Mariani et al, 2021)。

\section{2 结果}

\section{1 物种组成}

32 个采样点共采集到 27 种鱼类，其中舒溪采集 到23种，麻溪24种，浦溪22种。双因素交互相似性 分析发现, 物种组成随溪流(Global $R=0.36, P=$ 0.001 )与河段(Global $R=0.37, P=0.001$ )显著变化。 就溪流间物种组成变化而言, 舒溪与麻溪和浦溪间 均具有显著差异 (舒溪 $\times$ 麻溪: $R=0.31, P=0.035$; 舒溪 $\times$ 浦溪: $R=0.62, P=0.001)$, 而后两者间无 显著差异 $(R=0.16, P=0.15)$ 。由nMDS二维双标图 可见，舒溪鱼类群落明显区别于其他两条溪流，尤 其在中、下游河段(图2)。根据SIMPER结果，光唇 鱼(Acrossocheilus fasciatus)、原缨口鳅(Van manenia stenosoma)、稀有花鳅(Cobitis rarus)在舒溪中的多 度较高，但在麻溪和浦溪中多度明显下降; 相反, 河川沙塘鳢 (Odontobutis obscurus)、麦穗鱼 (Pseudorasbora parva) 等物种在麻溪和浦溪中的多 度较高, 而在舒溪中较低(表2)。

\section{2 功能 $\alpha$ 多样性}

双因素方差分析显示：FRic和FDiv随溪流显著 变化, FRic在舒溪(3.73 \pm 1.23)显著低于麻溪(9.13 \pm

表1 本研究所使用的鱼类功能特征

Table 1 The functional traits measured for each fish species in this study

\begin{tabular}{|c|c|c|c|}
\hline 功能特征 Functional traits & 计算方法 Measure & 生态学意义 Ecological meaning & 参考文献 Reference \\
\hline 眼睛相对大小 Relative eye size & 眼径/头长 $\mathrm{Ed} / \mathrm{Hd}$ & 对食物的可视范围 Visual scope to food & Boyle \& Horn, 2006 \\
\hline 眼睛相对位置 Relative eye position & 眼位/头高 $E h / H d$ & 在水体中的垂直位置 Vertical position in water & Gatz, 1979 \\
\hline 口裂位置 Mouth gape position & 口位/头高 $\mathrm{Mo} / \mathrm{Hd}$ & 在水中的生活位置 Living position in water & Sibbing \& Nagelkerke, 2000 \\
\hline 相对肠长 Relative gut length & 肠长/体长 $\mathrm{Gl} / \mathrm{Bl}$ & 食物消化能力 Ability to digest food & Kramer \& Bryant, 1995 \\
\hline 体型指标I Body shape I & 体高/体宽 $B d / B w$ & $\begin{array}{l}\text { 在水体中的栖息位置及游泳能力 Habitat location } \\
\text { and swimming ability in water }\end{array}$ & Sibbing \& Nagelkerke, 2000 \\
\hline 体型指标II Body shape II & 体高/体长 $B d / B l$ & $\begin{array}{l}\text { 在水体中的栖息位置及游泳能力 Habitat location } \\
\text { and swimming ability in water }\end{array}$ & Sibbing \& Nagelkerke, 2000 \\
\hline 尾柄对游泳的贡献 & 最大尾鯺高/最小尾柄宽 & 游泳持久性 & Webb, 1984 \\
\hline $\begin{array}{l}\text { Contribution of caudal peduncle to the } \\
\text { swimming capability }\end{array}$ & $C F d / C P d$ & Staying power to swimming & \\
\hline
\end{tabular}

Ed, Eye diameter; Eh, Eye height; $H d$, Head depth; Mo, Mouth orientation; Gl, Gut length; Bl, Body length; Bd, Body depth; Bw, Body width; $C F d$, Maximum caudal fin depth; $C P d$, Caudal peduncle depth. 
1.74)和浦溪 $(10.57 \pm 2.55)(P<0.05)$, 而FDiv在舒 溪 $(0.82 \pm 0.11)$ 中显著高于麻溪 $(0.75 \pm 0.01)$ 和浦溪 $(0.76 \pm 0.01)(P<0.05)$; 仅FRic随河段显著变化, 上游的FRic显著低于中游和下游; FRic 和FDiv受河 流和河段的显著性交互影响, FEve不随河流和河段 显著变化(表3)。

在SPSS软件中运用曲线估计解析鱼类功能多 样性与距河源距离的关系, 结果显示: 舒溪中FRic 与距河源距离呈显著线性相关，从上游至下游逐渐 增大 $(P<0.05) ;$ FRic 在麻溪和浦溪中与距河源距离 呈二项式关系, 从上游至下游先增大后减小, 呈钟 形分布 $(P<0.05) ; F E v e$ 仅在麻溪中与距河源距离显 著相关(正线性关系) $(P<0.1)$; 舒溪中FDiv与距河 源距离呈显著线性相关, 从上游至下游逐渐减小, FDiv在浦溪中与距河源距离呈显著的二项式关系, 从上游先降低后上升 $(P<0.1)$ (图3, 表4)。

\section{3 功能 $\beta$ 多样性}

3 条溪流的功能 $\beta$ 多样性平均值分别为 $0.53 \pm$ 0.03 (舒溪)、 $0.75 \pm 0.03$ (麻溪)和 $0.71 \pm 0.05$ (浦溪), 其中, 舒溪的功能 $\beta$ 多样性主要由嵌套成分贡献, 占比高达 $75.47 \%$, 而在麻溪和浦溪中, 嵌套与周转 成分对功能 $\beta$ 多样性的贡献相对接近(麻溪: 嵌套和 周转分别占 $45.33 \%$ 和 $54.37 \%$; 浦溪: 嵌套和周转分 别占 $57.75 \%$ 和 $42.25 \%)$ 。

根据双因素方差分析，功能 $\beta$ 多样性在溪流间
有显著差异 $(P<0.05)$, 舒溪的 $\beta s ø r$ 显著低于浦溪和 麻溪 $(P<0.05)$; 尽管嵌套成分 $\beta n e s$ 不随溪流显著变 化, 但周转成分 $\beta t u r$ 在舒溪中显著低于浦溪和麻溪 $(P<0.05)$ 。功能 $\beta$ 多样性及其成分均不随河段显著 变化 $(P>0.1)$ 。此外, $\beta s ø r$ 和 $\beta$ tur 受溪流与河段的交 互影响 $(P<0.1)$ (表3)。

曲线估计显示, 仅 $\beta s ø r$ 与距河源距离显著相关 $(P<0.1)$, 其中在舒溪中呈线性相关, 从上游至下 游呈下降趋势; 在麻溪中呈二项式U型分布, 从上 游至下游先下降后上升, 但在浦溪中与距河源距离

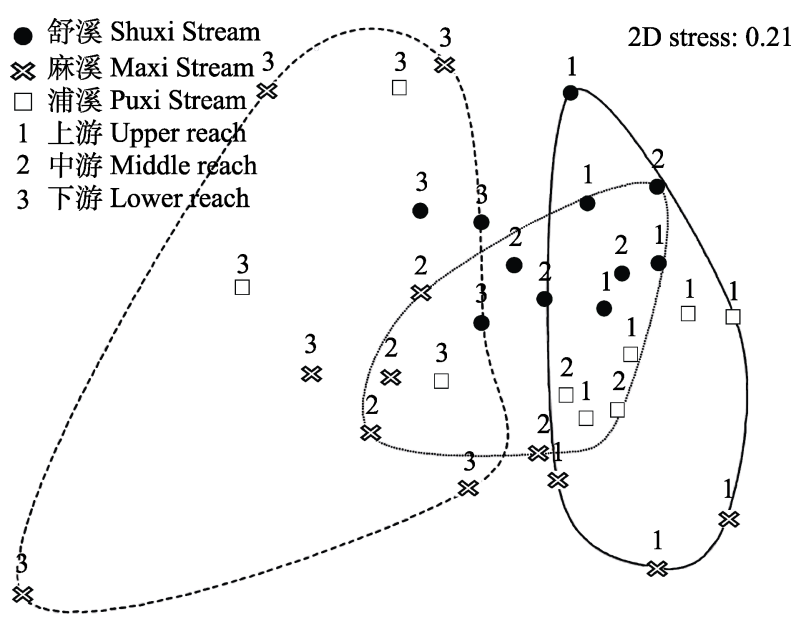

图2 溪流间鱼类物种组成结构的非度量多维标度法双标图 Fig. 2 Spatial variations in species composition of fishes across streams and reaches based on nMDS

表2 基于相似百分比分析得到的溪流间物种组成差异的鱼类关键种

Table 2 The key fish species contributing the between-stream composition difference based on similarity percentages

\begin{tabular}{|c|c|c|c|c|c|c|c|}
\hline \multirow[t]{2}{*}{ 物种 Species } & \multicolumn{3}{|c|}{ 平均多度 Average abundance } & \multicolumn{2}{|c|}{ 舒溪 vs. 麻溪 Shuxi vs. Maxi } & \multicolumn{2}{|c|}{ 舒溪 vs. 浦溪 Shuxi vs. Puxi } \\
\hline & $\begin{array}{l}\text { 舒溪 } \\
\text { Shuxi }\end{array}$ & $\begin{array}{l}\text { 麻溪 } \\
\text { Maxi }\end{array}$ & $\begin{array}{l}\text { 浦溪 } \\
\text { Puxi }\end{array}$ & $\begin{array}{l}\text { 平均不相似性 } \\
\text { Average dissimilarity }\end{array}$ & $\begin{array}{l}\text { 贡献率 } \\
\text { Contribution (\%) } \\
\end{array}$ & $\begin{array}{l}\text { 平均不相似性 } \\
\text { Average dissimilarity }\end{array}$ & $\begin{array}{l}\text { 贡献率 } \\
\text { yContribution (\%) }\end{array}$ \\
\hline 光唇鱼 Acrossocheilus fasciatus & 0.91 & 0.17 & & 4.69 & 8.27 & & \\
\hline 原缨口鳅 Vanmanenia stenosoma & 0.91 & 0.33 & 0 & 4.01 & 7.08 & 5.09 & 10.33 \\
\hline 稀有花鳅 Cobitis rarus & 0.73 & 0.17 & 0.11 & 3.95 & 6.96 & 3.74 & 7.59 \\
\hline 河川沙塘鳢 Odontobutis obscurus & 0 & 0.58 & & 3.34 & 5.89 & & \\
\hline 麦穗鱼 Pseudorasbora parva & 0.27 & & 0.56 & & & 2.84 & 5.77 \\
\hline 中华花鳅 Cobitis sinensis & 0.55 & 0.17 & 0.56 & 3.28 & 5.79 & 2.83 & 5.75 \\
\hline 高体鳑鲏 Rhodeus ocellatus & 0.55 & 0.58 & 0.56 & 3.05 & 5.37 & 2.82 & 5.72 \\
\hline 花斑副沙鳅 Parabotia fasciata & 0.27 & & 0.44 & $V$ & & 2.75 & 5.58 \\
\hline 似䱻 Belligobio nummifer & 0.45 & 0.33 & 0.33 & 2.93 & 5.17 & 2.73 & 5.54 \\
\hline 泥鳅 Misgurnus anguillicaudatus & 0.64 & 0.67 & & 2.87 & 5.07 & & \\
\hline 银鮈 Squalidus argentatus & 0.36 & 0.17 & 0.44 & 2.54 & 4.47 & 2.73 & 5.54 \\
\hline
\end{tabular}


表3 溪流和河段对鱼类功能 $\alpha$ 多样性和 $\beta$ 多样性的影响

Table 3 Effect of streams and segments on the functional $\alpha$ and $\beta$ diversity of fish assemblages

\begin{tabular}{|c|c|c|c|c|c|c|}
\hline $\begin{array}{l}\text { 功能多样性 } \\
\text { Functional diversity }\end{array}$ & $\begin{array}{l}\text { 多样性指数 } \\
\text { Diversity index }\end{array}$ & 分类变量 Factors & T-III SS & $F$ & $P$ & 组间比较 S-N-K \\
\hline \multirow{9}{*}{$\begin{array}{l}\alpha \text { 多样性 } \\
\alpha \text { diversity }\end{array}$} & \multirow{3}{*}{$\begin{array}{l}\text { 功能丰富度 } \\
\text { Functional richness }\end{array}$} & 溪流 Stream & 268.569 & 5.629 & 0.010 & 舒溪 $<$ 麻溪 $=$ 浦溪 $S S<M S=P S$ \\
\hline & & 河段 Segment & 173.811 & 3.643 & 0.042 & 上游 $<$ 中游 $=$ 下游 $U p<M i=L o$ \\
\hline & & 溪流 $\times$ 河段 Stream $\times$ Segment & 300.09 & 3.145 & 0.034 & \\
\hline & \multirow{3}{*}{$\begin{array}{l}\text { 功能均匀度 } \\
\text { Functional evenness }\end{array}$} & 溪流 Stream & 0.003 & 1.444 & 0.256 & \\
\hline & & 河段 Segment & 0.002 & 1.033 & 0.372 & \\
\hline & & 溪流 $\times$ 河段 Stream $\times$ Segment & 0.007 & 1.485 & 0.239 & \\
\hline & \multirow{3}{*}{$\begin{array}{l}\text { 功能离散度 } \\
\text { Functional divergence }\end{array}$} & 溪流 Stream & 0.029 & 14.189 & 0.000 & 舒溪 $>$ 浦溪 $=$ 麻溪 $S S>P S=M S$ \\
\hline & & 河段 Segment & 0.001 & 0.658 & 0.527 & \\
\hline & & 溪流 $\times$ 河段 Stream $\times$ Segment & 0.011 & 2.595 & 0.063 & \\
\hline \multirow{9}{*}{$\begin{array}{l}\beta \text { 多样性 } \\
\beta \text { diversity }\end{array}$} & \multirow{3}{*}{$\begin{array}{l}\text { 功能 } \beta \text { 多样性 } \\
\text { Functional } \beta \text { sør diversity }\end{array}$} & 溪流 Stream & 0.328 & 13.749 & 0.000 & 舒溪 $<$ 浦溪 $=$ 麻溪 $S S<P S=M S$ \\
\hline & & 河段 Segment & 0.003 & 0.125 & 0.883 & \\
\hline & & 溪流 $\times$ 河段 Stream $\times$ Segment & 0.086 & 1.804 & 0.063 & \\
\hline & \multirow{3}{*}{$\begin{array}{l}\text { 功能嵌套 } \\
\text { Functional } \beta \text { nes diversity }\end{array}$} & 溪流 Stream & 0.029 & 1.661 & 0.212 & \\
\hline & & 河段 Segment & 0.007 & 0.385 & 0.685 & \\
\hline & & 溪流 $\times$ 河段 Stream $\times$ Segment & 0.014 & 0.385 & 0.817 & \\
\hline & \multirow{3}{*}{$\begin{array}{l}\text { 功能周转 } \\
\text { Functional } \beta \text { tur diversity }\end{array}$} & 溪流 Stream & 0.477 & 25.032 & 0.000 & \multirow[t]{3}{*}{ 舒溪 $<$ 浦溪 $<$ 麻溪 $S S<P S<M S$} \\
\hline & & 河段 Segment & 0.013 & 0.695 & 0.509 & \\
\hline & & 溪流 $\times$ 河段 Stream $\times$ Segment & 0.091 & 2.400 & 0.079 & \\
\hline
\end{tabular}

SS, MS and PS represent Shuxi, Maxi and Puxi Stream, respectively. Up, Mi and Lo represent the upper, middle and lower segments, respectively.

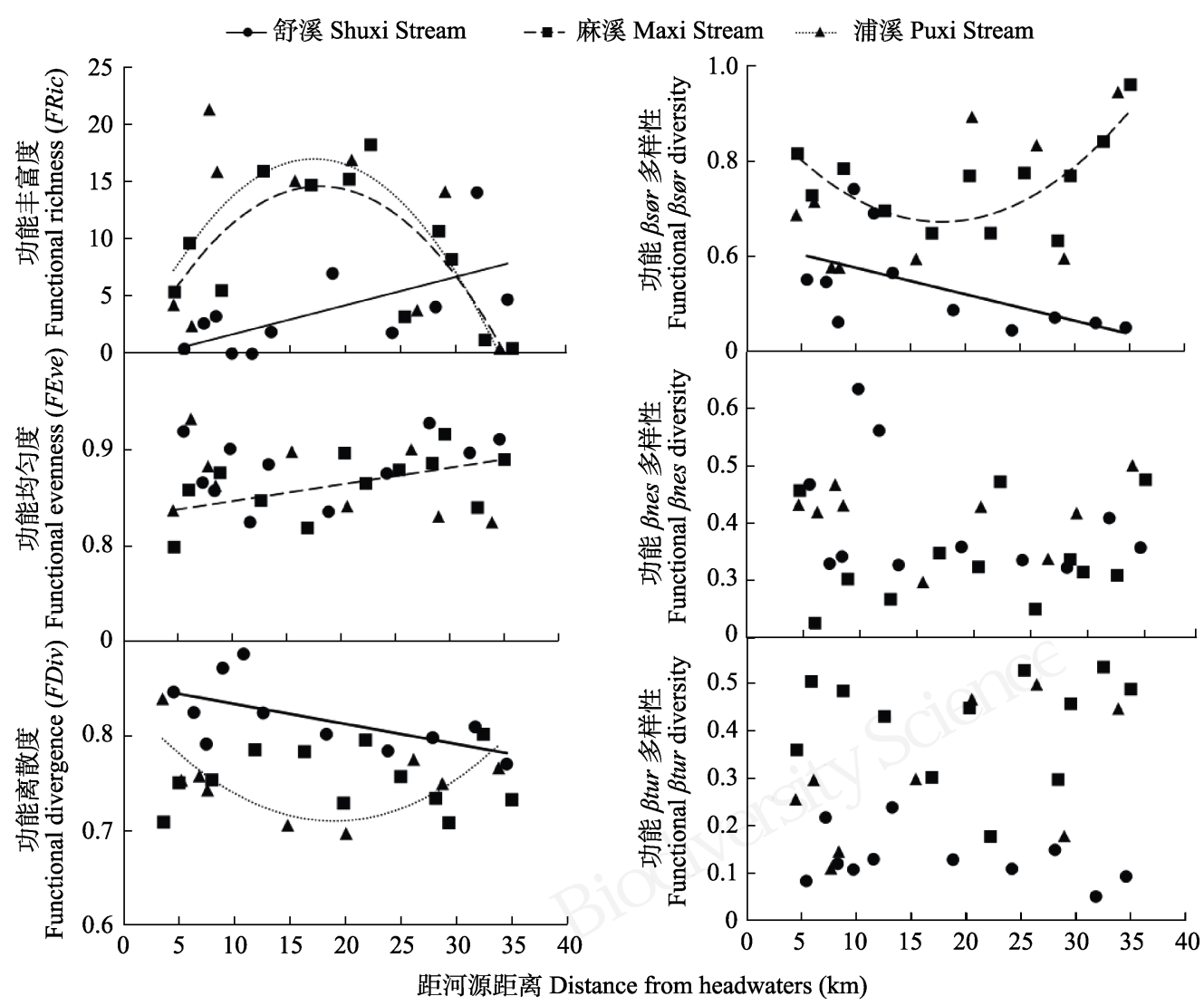

图3 功能多样性与距河源距离的关系

Fig. 3 Relationship between the functional diversity and the distance from headwaters 
表4 功能 $\alpha$ 和 $\beta$ 多样性与距河源距离的线性和二次项模型的统计数据

Table 4 Summary statistic for the linear and quadratic models explaining the correlations between functional diversities and distance from headwaters

\begin{tabular}{|c|c|c|c|c|c|c|}
\hline 功能多样性 & 多样性指数 & 溪流 & 模型 & $R^{2}$ & $F$ & $P$ \\
\hline Functional diversity & Diversity index & Stream & Models & & & \\
\hline \multirow[t]{18}{*}{ $\alpha$ 多样性 $\alpha$ diversity } & \multirow{6}{*}{$\begin{array}{l}\text { 功能丰富度 } \\
\text { Functional richness }\end{array}$} & \multirow[t]{2}{*}{ 舒溪 Shuxi } & 线性 Linear & 0.410 & 6.310 & $0.033^{* \#}$ \\
\hline & & & 二次项 Quadratic & 0.418 & 2.870 & 0.115 \\
\hline & & \multirow[t]{2}{*}{ 麻溪 Maxi } & 线性 Linear & 0.092 & 1.013 & 0.338 \\
\hline & & & 二次项 Quadratic & 0.648 & 8.288 & $0.009 *$ \\
\hline & & \multirow[t]{2}{*}{ 浦溪 Puxi } & 线性 Linear & 0.052 & 0.387 & 0.554 \\
\hline & & & 二次项 Quadratic & 0.434 & 2.304 & 0.081 \\
\hline & \multirow{6}{*}{$\begin{array}{l}\text { 功能均匀度 } \\
\text { Functional evenness }\end{array}$} & \multirow[t]{2}{*}{ 舒溪 Shuxi } & 线性 Linear & 0.120 & 1.210 & 0.300 \\
\hline & & & 二次项 Quadratic & 0.350 & 2.180 & 0.175 \\
\hline & & \multirow[t]{2}{*}{ 麻溪 Maxi } & 线性 Linear & 0.288 & 4.045 & $0.072^{\#}$ \\
\hline & & & 二次项 Quadratic & 0.317 & 2.091 & 0.182 \\
\hline & & \multirow[t]{2}{*}{ 浦溪 Puxi } & 线性 Linear & 0.183 & 1.571 & 0.250 \\
\hline & & & 二次项 Quadratic & 0.274 & 1.132 & 0.383 \\
\hline & \multirow{6}{*}{$\begin{array}{l}\text { 功能离散度 } \\
\text { Functional divergence }\end{array}$} & \multirow[t]{2}{*}{ 舒溪 Shuxi } & 线性 Linear & 0.395 & 5.880 & $0.038^{* \#}$ \\
\hline & & & 二次项 Quadratic & 0.398 & 2.640 & 0.132 \\
\hline & & \multirow[t]{2}{*}{ 麻溪 Maxi } & 线性 Linear & 0.003 & 0.029 & 0.868 \\
\hline & & & 二次项 Quadratic & 0.132 & 0.682 & 0.530 \\
\hline & & \multirow[t]{2}{*}{ 浦溪 Puxi } & 线性 Linear & 0.045 & 0.328 & 0.585 \\
\hline & & & 二次项 Quadratic & 0.578 & 4.104 & 0.075 \\
\hline \multirow[t]{18}{*}{$\beta$ 多样性 $\beta$ diversity } & \multirow{6}{*}{$\begin{array}{l}\text { 功能 } \beta s ø r \text { 多样性 } \\
\text { Functional } \beta s ø r \text { diversity }\end{array}$} & \multirow[t]{2}{*}{ 舒溪 Shuxi } & 线性 Linear & 0.339 & 4.624 & $0.060^{\#}$ \\
\hline & & & 二次项 Quadratic & 0.347 & 2.123 & 0.182 \\
\hline & & \multirow[t]{2}{*}{ 麻溪 Maxi } & 线性 Linear & 0.080 & 0.865 & 0.374 \\
\hline & & & 二次项 Quadratic & 0.598 & 6.700 & $0.017 *$ \\
\hline & & \multirow[t]{2}{*}{ 浦溪 Puxi } & 线性 Linear & 0.324 & 3.356 & 0.110 \\
\hline & & & 二次项 Quadratic & 0.352 & 1.632 & 0.272 \\
\hline & \multirow{6}{*}{$\begin{array}{l}\text { 功能 } \beta \text { nes多样性 } \\
\text { Functional } \beta \text { nes diversity }\end{array}$} & \multirow[t]{2}{*}{ 舒溪 Shuxi } & 线性 Linear & 0.124 & 1.278 & 0.287 \\
\hline & & & 二次项 Quadratic & 0.132 & 0.610 & 0.567 \\
\hline & & \multirow[t]{2}{*}{ 麻溪 Maxi } & 线性 Linear & 0.039 & 0.407 & 0.538 \\
\hline & & & 二次项 Quadratic & 0.102 & 0.508 & 0.618 \\
\hline & & \multirow[t]{2}{*}{ 浦溪 Puxi } & 线性 Linear & 0.000 & 0.002 & 0.962 \\
\hline & & & 二次项 Quadratic & 0.477 & 2.737 & 0.431 \\
\hline & \multirow{6}{*}{$\begin{array}{l}\text { 功能 } \beta \text { tur多样性 } \\
\text { Functional } \beta \text { tur diversity }\end{array}$} & \multirow[t]{2}{*}{ 舒溪 Shuxi } & 线性 Linear & 0.147 & 1.551 & 0.244 \\
\hline & & & 二次项 Quadratic & 0.255 & 1.368 & 0.308 \\
\hline & & \multirow[t]{2}{*}{ 麻溪 Maxi } & 线性 Linear & 0.008 & 0.079 & 0.785 \\
\hline & & & 二次项 Quadratic & 0.186 & 1.029 & 0.396 \\
\hline & & \multirow[t]{2}{*}{ 浦溪 Puxi } & 线性 Linear & 0.322 & 3.320 & 0.111 \\
\hline & & & 二次项 Quadratic & 0.338 & 1.533 & 0.290 \\
\hline
\end{tabular}

$* P<0.05$; \# 线性关系 Linear relationship

无显著相关。Bnes和 $\beta$ tur 与距河源距离无显著的线 性或二项式关系 $(P>0.10)$ (表3，图3)。

\section{3 讨论}

沿着上游-下游的纵向梯度, 溪流系统存在显 著的空间异质性, 栖息地多样化且具有一定的复杂 性，进而影响鱼类物种组成及其数量的分布 (Suvarnaraksha et al, 2012)。此外, 在河流网络结构
中, 不同河段在河网中的空间位置不同，其鱼类的 迁入迁出速率也存在差异, 因而其鱼类多样性也可 能存在差异(Yan et al, 2011)。本研究发现，在人类干 扰程度较轻的舒溪中, 鱼类功能丰富度与距河源距 离呈线性正相关, 反映了功能丰富度从上游至下游 呈线性上升趋势，该结果与 $\mathrm{Li}$ 等(2018)所观察到的 物种丰富度的纵向梯度规律一致。关于生物多样性 和生态系统功能的经典研究表明, 高物种多样性往 
往产生高功能多样性(Tilman et al, 1997); 同时, 本 研究结果也进一步证实了河流连续体(The River Continuum Concept)的预期, 即: 从河流上游至下 游, 随着资源多样性的增加, 鱼类营养多样性上升, 最终流域下游的功能多样性增加 (Thorp et al, 2006)。但是, 本研究也发现, 舒溪鱼类功能离散度 (FDiv)的纵向梯度分布规律与FRic相反一从上游 至下游呈线性下降趋势。FDiv衡量群落功能性状多 度分布的最大离散程度, 离散度高表明物种间的生 态位分化程度高、资源竞争程度低(Mason et al, 2005)。有研究表明, 溪流系统的栖息地多样性、复 杂性和稳定性从上游至下游显著变化。同溪流下游 相比, 上游河段的水文和栖息地条件的季节变化非 常大, 因而鱼类的种群数量波动较大, 通常达不到 形成种间甚至种内竞争的种群数量; 相反, 下游河 段的水文和栖息地条件的稳定性较高, 鱼类的种群 数量也相应较大, 种内竞争激烈(Horwitz, 1978)。据 此, 我们推测, 溪流环境稳定性的空间变化可能影 响了溪流鱼类的种群数量及种间资源竞争强度变 化, 进而可能促进了鱼类功能离散度由上游至下游 逐渐减小这一空间格局。

本研究还发现, 在受干扰程度较低的舒溪, 从 上游至下游, 鱼类功能 $\beta$ 多样性 $(\beta s ø r)$ 呈线性下降趋 势, 该空间格局与功能丰富度正好相反。类似结果 也见于 $\mathrm{Li}$ 等(2018)针对物种多样性的研究结果。众 所周知, $\alpha$ 多样性和 $\beta$ 多样性的内涵不同(Kessler et al, 2009), 正因为两者的生物学意义差异, $\alpha$ 多样性和 $\beta$ 多样性沿着环境梯度的空间格局可能不一致甚至 相反(Tylianakis et al, 2005)。同中下游河段相比, 上 游河段的环境条件苛刻(如低营养、低温、急流等), 尽管鱼类物种较少但其特有性较高, 因而具有极高 的 $\beta$ 多样性(Li et al, 2018; 张东等, 2018)。因此, 有理 由相信, 若上游河段特有鱼类物种也具有特殊的功 能特征, 那么, 上游河段的鱼类功能 $\beta$ 多样性也就 相对较高, 这在一定程度上能够解释本研究所观察 到的舒溪鱼类功能 $\beta$ 多样性为何从上游至下游呈下 降趋势。此外, 本研究还发现, 在舒溪中, 鱼类功能 $\beta$ 多样性主要由嵌套成分贡献, 其比例超过 $3 / 4$ 。从 一个群落到另一个群落, 其物种/功能的变化模式 包括物种/功能丢失与物种/功能获得(Villéger et al, 2014), 若丢失和获得过程两者兼有则呈群落周转 格局, 若仅含丢失或获得 1 个过程则呈群落嵌套格
局(Leprieur et al, 2011)。有研究发现, 沿着特定的环 境梯度, 周转和嵌套成分对 $\beta$ 多样性的贡献大小, 会 随物种和功能多样性而变化一一物种 $\beta$ 多样性主要 由周转主导, 而功能 $\beta$ 多样性由嵌套主导(Bishop et al, 2014; 张东等, 2018)。

本研究中的3条河源溪流的人为干扰强度不同, 同舒溪相比, 麻溪和浦溪的人为干扰强度明显较重 (附录1), 且同一溪流内人为扰动强度由上游(高坡) 向下游(低坡)逐渐增大(附录2)。FRic和FDiv两个指 数受溪流和河段 2 个因素的交互影响, 这反映了人 类干扰对鱼类功能 $\alpha$ 多样性的影响视不同河段而变 化。我们发现, 同舒溪相比, 麻溪和浦溪的鱼类物 种组成发生了显著变化, 主要表现为光唇鱼、原缨 口鳅、稀有花鳅等物种的减少或丢失以及河川沙塘 鳢和麦穗鱼等物种的增多或获得。根据Chu等(2015) 和Liu等(2019), 上述丢失的光唇鱼、原缨口鳅和稀 有花鱾为研究区域内河源溪流中的土著种, 而获得 的河川沙塘鳢和麦穗鱼原生活于研究区域内的低 地水体, 故在河源溪流中隶属本地入侵种。溪流间 各个河段的物种组成均有差异, 尤其是以下游更为 明显(图2), 这也意味着上述土著物种丢失和本地入 侵种获得过程在各个河段均有发生。麻溪和浦溪的 中上游河段的FRic 高于舒溪, 但其下游的FRic 却低 于舒溪(图3)。我们认为, 因中上游河段的人为干扰 强度相对较小, 麻溪和浦溪中上游河段的鱼类物种 组成变化可能是以本地入侵种的获得为主导(较轻 的人为干扰可能没有导致土著种的大量丢失), 那 么, 当获得的本地入侵种带来一定的新功能特征时, 将提高麻溪和浦溪中上游河段的功能丰富度。但是, 在下游河段, 因人为干扰强度较大, 其物种组成变 化不仅体现在本地入侵种的获得, 也表现为很多土 著种的丢失, 那么, 当获得物种所带来的新功能特 征远少于丢失物种所具有的功能特征时, 将导致麻 溪和浦溪下游河段功能丰富度的下降。我们还发现, 在麻溪和浦溪上中游, 随着人为干扰增大, 功能离 散度逐渐降低。我们认为, 在人类活动的影响下, 浦溪和麻溪中上游河段获得了一些本地入侵种, 这 些本地入侵种在食物等资源利用上与土著鱼类存 在较高的重叠。朱仁等(2012)通过胃含物分析发现, 在青江陈村水库上游河源溪流中, 以本地入侵种 麦穗鱼为例, 其与光唇鱼、宽鯺鱲(Zacco platypus) 等土著种的食物重叠系数超过 $90 \%$; 相反, 以土著 
物种吻虾虎鱼为例, 其与光唇鱼、宽鯺鱲等其他土 著种的食物重叠系数低于50\%。这可能意味着, 在 浦溪和麻溪中上游河段, 因本地入侵种获得所带来 的种间资源分化下降、种间竞争加剧, 导致了其鱼 类功能离散度相对下降。

物种取代过程既能够驱动基于物种组成的分 类同质化/异质化过程, 也能够带来基于物种特征 的功能同质化/异质化变化(Su et al, 2015)。同舒溪 相比, 麻溪和浦溪的平均功能 $\beta$ 多样性 $(\beta s ø r)$ 显著上 升, 由此可见, 人类干扰促进了溪流鱼类群落趋于 功能异质化。考虑到同一溪流不同河段间人类干扰 强度存在差异, 当土著种和本地入侵种存在功能特 征上的差异时, 该物种异质化也将引起鱼类群落在 功能特征上趋于异质化。本研究还发现, 功能 $\beta s ø r$ 受溪流与河段的交互影响, 这意味着溪流间的 $\beta s ø r$ 变化视河段而不同。在下游, 麻溪和浦溪的功能 $\beta$ 多样性相对于舒溪而言, 其变化程度增大, 这可以 通过人为干扰强度(如城市用地比例)沿纵向梯度的 空间分布来解释。尽管舒溪功能 $\beta$ 多样性主要由嵌 套成分组成(占比超过 $75 \%$ ), 麻溪和浦溪中嵌套成 分所占比例明显下降 (麻溪： 57.75\%; 浦溪： 45.33\%)。若环境变化具有连续性, 则多为嵌套模式; 相反, 若环境变化呈间断性, 则主要表现为周转模 式(Roberts \& Hitt, 2010)。根据“河流连续体概念”, 河流系统具有连续性、等级性和异质性等多重属性; 从河源至河口, 其非生物因子及生物成分的空间变 化具有连续性特点(Vannote et al, 1980)。但是, 受水 坝、土地利用等人类活动的影响, 很多河流的空间 连续性被打断, 呈现为 “序列不连续”特点(Ward \& Stanford, 1983)。因此, 同舒溪相比, 麻溪和浦溪中 较强的土地利用(陆君等, 2014)和水污染排放(李响 等, 2014)可能增大了其序列不连续, 进而导致鱼类 群落及其功能特征的周转率上升。

\section{ORCID}

贺佳云 (D) https://orcid.org/0000-0003-2221-3501

张东 (D) https://orcid.org/0000-0002-4265-1077

严云志 (D) https://orcid.org/0000-0001-8088-3867

\section{参考文献}

Allan JD (2004) Landscapes and riverscapes: The influence of land use on stream ecosystems. Annual Review of Ecology, Evolution, and Systematics, 35, 257-284.
Baselga A (2010) Partitioning the turnover and nestedness components of beta diversity. Global Ecology and Biogeography, 19, 134-143.

Bishop TR, Robertson MP, van Rensburg BJ, Parr CL (2014) Elevation-diversity patterns through space and time: Ant communities of the Maloti-Drakensberg Mountains of southern Africa. Journal of Biogeography, 41, 2256-2268.

Boyle KS, Horn MH (2006) Comparison of feeding guild structure and ecomorphology of intertidal fish assemblages from central California and central Chile. Marine Ecology Progress Series, 319, 65-84.

Chen XY (2013) Checklist of fishes of Yunnan. Zoological Research, 34, 281-343. (in Chinese with English abstract) [陈小勇 (2013) 云南鱼类名录. 动物学研究, 34, 281343.]

Chu L, Wang WJ, Zhu R, Yan YZ, Chen YF, Wang LZ (2015) Variation in fish assemblages across impoundments of low-head dams in headwater streams of the Qingyi River, China: Effects of abiotic factors and native invaders. Environmental Biology of Fishes, 98, 101-112.

Ding N, Yang WF, Zhou YC, González-Bergonzoni I, Zhang J, Chen K, Vidal N, Jeppesen E, Liu ZW, Wang BX (2017) Different responses of functional traits and diversity of stream macroinvertebrates to environmental and spatial factors in the Xishuangbanna watershed of the upper Mekong River Basin, China. Science of the Total Environment, 574, 288-299.

Dudgeon D, Arthington AH, Gessner MO, Kawabata ZI, Knowler DJ, Lévêque C, Naiman RJ, Prieur-Richard AH, Soto D, Stiassny MLJ, Sullivan CA (2006) Freshwater biodiversity: Importance, threats, status and conservation challenges. Biological Reviews, 81, 163-182.

Dumay O, Tari PS, Tomasini JA, Mouillot D (2004) Functional groups of lagoon fish species in Languedoc Roussillon, southern France. Journal of Fish Biology, 64, 970-983.

Gatz AJ Jr (1979) Community organization in fishes as indicated by morphological features. Ecology, 60, 711-718.

Göthe E, Baattrup-Pedersen A, Wiberg-Larsen P, Graeber D, Kristensen EA, Friberg N (2017) Environmental and spatial controls of taxonomic versus trait composition of stream biota. Freshwater Biology, 62, 397-413.

Horwitz RJ (1978) Temporal variability patterns and the distributional patterns of stream fishes. Ecological Monographs, 48, 307-321.

Kessler M, Abrahamczyk S, Bos M, Buchori D, Putra DD, Gradstein SR, Höhn P, Kluge J, Orend F, Pitopang R, Saleh S, Schulze CH, Sporn SG, Steffan-Dewenter I, Tjitrosoedirdjo SS, Tscharntke T (2009) Alpha and beta diversity of plants and animals along a tropical land-use gradient. Ecological Applications, 19, 2142-2156.

Kramer DL, Bryant MJ (1995) Intestine length in the fishes of a tropical stream. 2. Relationships to diet-the long and short of a convoluted issue. Environmental Biology of 
Fishes, 42, 129-141.

Laliberté E, Legendre P (2010) A distance-based framework for measuring functional diversity from multiple traits. Ecology, 91, 299-305.

Leprieur F, Tedesco PA, Hugueny B, Beauchard O, Dürr HH, Brosse S, Oberdorff T (2011) Partitioning global patterns of freshwater fish beta diversity reveals contrasting signatures of past climate changes. Ecology Letters, 14, 325-334.

Li X, Lu J, Qian ML, Wang XR, Fan ZQ, Wang SB (2014) Study on pollution loading and water environmental capacity in watershed-A case study of Taiping Lake Basin, Anhui Province, China. China Environmental Science, 34, 2063-2070. (in Chinese with English abstract) [李响, 陆君, 钱敏蕾, 王祥荣, 㚞正球, 王寿兵 (2014) 流域污染负荷 解析与环境容量研究——安徽太平湖流域为例. 中国 环境科学, 34, 2063-2070.]

Li YR, Tao J, Chu L, Yan YZ (2018) Effects of anthropogenic disturbances on $\alpha$ and $\beta$ diversity of fish assemblages and their longitudinal patterns in subtropical streams, China. Ecology of Freshwater Fish, 27, 433-441.

Li ZF, Wang J, Liu ZY, Meng XL, Heino J, Jiang XK, Xiong X, Jiang XM, Xie ZC (2019) Different responses of taxonomic and functional structures of stream macroinvertebrate communities to local stressors and regional factors in a subtropical biodiversity hotspot. Science of the Total Environment, 655, 1288-1300.

Lin L, Soni A, Sabik LM, Drake C (2020) Early- and late-stage cancer diagnosis under 3 years of Medicaid expansion. American Journal of Preventive Medicine, 60, 104-109.

Liu Y, Wang YR, Zhu Q, Li YR, Kang B, Chu L, Yan YZ (2019) Effects of low-head dams on fish assemblages in subtropical streams: Context dependence on species category and data type. River Research and Applications, 35, 396-404.

Lu J, Liu YF, Huang HH, Qi K, Li X, Fan ZQ (2014) Correlation analysis between land use structure and water quality of the Taiping Lake watershed in Huangshan. Journal of Fudan University (Natural Science), 53, 731-736, 746. (in Chinese with English abstract) [陆君, 刘亚风, 黄 洪辉, 齐珂, 李响, 㚞正球 (2014) 黄山市太平湖流域土 地利用结构与河流水质相关性分析. 复旦学报(自然科学 版), 53, 731-736, 746.]

Mariani JJ, Pavlicova M, Choi CJ, Basaraba C, Carpenter KM, Mahony AL, Brooks DJ, Bisaga A, Naqvi N, Nunes EV, Levin FR (2021) Quetiapine treatment for cannabis use disorder. Drug and Alcohol Dependence, 218, 108366.

Mason NWH, Mouillot D, Lee WG, Wilson JB (2005) Functional richness, functional evenness and functional divergence: The primary components of functional diversity. Oikos, 111, 112-118.

Mazzoni R, Lobón-Cerviá J (2008) Longitudinal structure, density and production rates of a neotropical stream fish assemblage: The river Ubatiba in the Serra do Mar, southeast Brazil. Ecography, 23, 588-602.

Mouchet MA, Villéger S, Mason NWH, Mouillot D (2010) Functional diversity measures: An overview of their redundancy and their ability to discriminate community assembly rules. Functional Ecology, 24, 867-876.

Münkemüller T, de Bello F, Meynard CN, Gravel D, Lavergne S, Mouillot D, Mouquet N, Thuiller W (2012) From diversity indices to community assembly processes: A test with simulated data. Ecography, 35, 468-480.

Nelson JS (2006) Fishes of the World, 4th edn. John Wiley \& Sons, Hoboken, New Jersey.

Petchey OL, Gaston KJ (2006) Functional diversity: Back to basics and looking forward. Ecology Letters, 9, 741-758.

Poff LR, Ward JV (1990) Physical habitat template of lotic systems: Recovery in the context of historical pattern of spatiotemporal heterogeneity. Environmental Management, 14, 629-645.

Roberts JH, Hitt NP (2010) Longitudinal structure in temperate stream fish communities: Evaluating conceptual models with temporal data. American Fisheries Society Symposium, 73, 281-299.

Sax DF, Gaines SD (2003) Species diversity: From global decreases to local increases. Trends in Ecology \& Evolution, 18, 561-566.

Sibbing FA, Nagelkerke LAJ (2000) Resource partitioning by Lake Tana barbs predicted from fish morphometrics and prey characteristics. Reviews in Fish Biology and Fisheries, 10, 393-437.

Soininen J, Heino J, Wang JJ (2018) A meta-analysis of nestedness and turnover components of beta diversity across organisms and ecosystems. Global Ecology and Biogeography, 27, 96-109.

Su GH, Xu J, Akasaka M, Molinos JG, Matsuzaki SIS (2015) Human impacts on functional and taxonomic homogenization of plateau fish assemblages in Yunnan, China. Global Ecology and Conservation, 4, 470-478.

Sui XY, Lu Z, Yan YZ, Chen YF, Jia YT (2014) Influence of a large dam on the longitudinal patterns of fish assemblages in Qingyi Stream. Zoological Research, 35, 362-372.

Suvarnaraksha A, Lek S, Lek-Ang S, Jutagate T (2012) Fish diversity and assemblage patterns along the longitudinal gradient of a tropical river in the Indo-Burma hotspot region (Ping-Wang River Basin, Thailand). Hydrobiologia, 694, 153-169.

Swenson NG, Anglada-Cordero P, Barone JA (2011) Deterministic tropical tree community turnover: Evidence from patterns of functional beta diversity along an elevational gradient. Proceedings of the Royal Society B: Biological Sciences, 278, 877-884.

Taylor CM, Warren ML Jr (2001) Dynamics in species composition of stream fish assemblages: Environmental variability and nested subsets. Ecology, 82, 2320-2330.

Thorp JH, Thoms MC, Delong MD (2006) The riverine 
ecosystem synthesis: Biocomplexity in river networks across space and time. River Research and Applications, 22, 123147.

Tilman D, Knops J, Wedin D, Reich P, Ritchie M, Siemann E (1997) The influence of functional diversity and composition on ecosystem processes. Science, 277, 1300-1302.

Tolonen KE, Leinonen K, Marttila H, Erkinaro J, Heino J (2017) Environmental predictability of taxonomic and functional community composition in high-latitude streams. Freshwater Biology, 62, 1-16.

Tylianakis JM, Klein AM, Tscharntke T (2005) Spatiotemporal variation in the diversity of Hymenoptera across a tropical habitat gradient. Ecology, 86, 3296-3302.

Vannote RL, Minshall GW, Cummins KW, Sedell JR, Cushing CE (1980) The river continuum concept. Canadian Journal of Fisheries and Aquatic Sciences, 37, 130-137.

Villéger S, Grenouillet G, Brosse S (2013) Decomposing functional $\beta$-diversity reveals that low functional $\beta$-diversity is driven by low functional turnover in European fish assemblages. Global Ecology and Biogeography, 22, 671-681.

Villéger S, Grenouillet G, Brosse S (2014) Functional homogenization exceeds taxonomic homogenization among European fish assemblages. Global Ecology and Biogeography, 23, 1450-1460.

Villéger S, Mason NWH, Mouillot D (2008) New multidimensional functional diversity indices for a multifaceted framework in functional ecology. Ecology, 89, 2290-2301.

Wang LZ, Lyons J, Kanehl P, Bannerman R (2001) Impacts of urbanization on stream habitat and fish across multiple spatial scales. Environmental Management, 28, 255-266.

Ward JV, Stanford JA (1983) The serial discontinuity concept of lotic ecosystems. Dynamics of Lotic Ecosystems.

Webb PW (1984) Body form, locomotion and foraging in aquatic vertebrates. American Zoologist, 24, 107-120.

Whittaker RH (1960) Vegetation of the Siskiyou Mountains, Oregon and California. Ecological Monographs, 30, 279338.

Yan YZ, Xiang XY, Chu L, Zhan YJ, Fu CZ (2011) Influences of local habitat and stream spatial position on fish assemblages in a dammed watershed, the Qingyi Stream, China. Ecology of Freshwater Fish, 20, 199-208.

Zhang D, Heng WJ, Chu L, Xu DP, Kang B, Yan YZ (2020) Taxonomic and functional diversity in a subtropical stream: A longitudinal pattern analysis. Ecology of Freshwater Fish, 29, 752-763.

Zhang D, Wan FY, Chu L, Yan YZ (2018) Longitudinal patterns in $\alpha$ and $\beta$ diversity of the taxonomic and functional organizations of stream fish assemblages in the Qingyi River. Biodiversity Science, 26, 1-13. (in Chinese with English abstract) [张东, 宛凤英, 储玲, 严云志 (2018) 青 七江鱼类分类群和功能群的 $\alpha$ 和 $\beta$ 多样性纵向梯度格局. 生物多样性, 26, 1-13.]

Zhu R, Yan YZ, Sun JJ, Huang HL, Tan XL, Lin L (2012) Feeding habits of fish assemblage in headwater streams of Chencun Reservoir in Huangshan Mountain. Chinese Journal of Ecology, 31, 359-366. (in Chinese with English abstract) [朱仁, 严云志, 孙建建, 黄海玲, 谈小龙, 林璐 (2012) 黄山陈村水库河源溪流鱼类群落的食性. 生态学 杂志, 31, 359-366.]

(责任编委: 陈小勇 责任编辑: 问文杰)

\section{附录 Supplementary Material}

\section{附录1 青弋江3条河源溪流每年排放的污染及不同土地利用比例的差异}

Appendix 1 Differences in the pollution emitted yearly and the proportions of land use across three headwaters of Qingyi River https://www.biodiversity-science.net/fileup/PDF/2020434-1.pdf

\section{附录2 不同坡度土地利用比例的变化}

Appendix 2 The variations in the proportion of land use across the areas of different slopes https://www.biodiversity-science.net/fileup/PDF/2020434-2.pdf

附录3 本研究功能 $\alpha$ 和 $\beta$ 多样性指数的计算公式

Appendix 3 The formulas of the functional $\alpha$ and $\beta$ diversity indices calculated in this study https://www.biodiversity-science.net/fileup/PDF/2020434-3.pdf 
贺佳云, 张东, 储玲, 严云志 (2021) 人为干扰对溪流鱼类功能多样性及其纵向梯度格局的影响. 生物多样性, 29,927-937. https://www.biodiversity-science.net/CN/10.17520/biods.2020434

附录1 青仜3条河源溪流每年排放的污染及不同土地利用比例的差异

Appendix 1 Differences in the pollution emitted yearly and the proportions of land use across three headwaters of Qingyi River

\begin{tabular}{llllll}
\hline $\begin{array}{l}\text { 人为干扰 } \\
\text { Disturbance }\end{array}$ & 变量类型 & 舒溪 & 麻溪 & 浦溪 & 参考文献 \\
Variable & Shuxi & Maxi & Puxi & Reference \\
\hline $\begin{array}{l}\text { 污染排放 Polluting emission } \\
\text { (t/year) }\end{array}$ & 化学需氧量 COD & 332.22 & $1,246.39$ & 998.41 & 李响等, 2014 \\
& 氨氮 $\mathrm{NH}_{3}$-N & 38.50 & 134.51 & 104.11 & \\
& 总磷 $\mathrm{TP}$ & 5.27 & 17.04 & 10.59 & \\
土地利用 Land use (\%) & 建筑用地 Urban land & 0.63 & 3.13 & 9.32 & \\
& 耕地 Farmland & 3.67 & 8.08 & 11.12 \\
& 林草地 Forest-grass land & 92.43 & 81.71 & 75.99 \\
& 其他用地 Others & 1.30 & 3.82 & 1.56 & \\
& & & & & \\
\hline
\end{tabular}

Li X, Lu J, Qian ML, Wang XR, Fan ZQ, Wang SB (2014) Study on pollution loading and water environmental capacity in watershed-A case study of Taiping Lake Basin, Anhui Province, China. China Environmental Science, 34, 2063-2070. (in Chinese with English abstract) [李响, 陆君, 钱敏蕾, 王祥荣, 樊正球, 王寿兵 (2014) 流域污染负荷解析与环境容量研究 ——安徽太平湖流域为例. 中国环境科学, 34, 2063-2070.]

Lu J, Liu YF, Huang HH, Qi K, Li X, Fan ZQ (2014) Correlation analysis between land use structure and water quality of the Taiping Lake watershed in Huangshan. Journal of Fudan University (Natural Science), 53, 731-736, 746. (in Chinese with English abstract) [陆君, 刘亚风, 黄洪辉, 齐珂, 李响, 焚正球 (2014) 黄山市太平湖流域土地利用结构与河流水质相关性 分析. 复旦学报(自然科学版), 53, 731-736, 746.] 
贺佳云, 张东, 储玲, 严云志 (2021) 人为干扰对溪流鱼类功能多样性及其纵向梯度格局的影响. 生物多样性, 29,927-937. https://www.biodiversity-science.net/CN/10.17520/biods.2020434

附录 2 不同坡度土地利用比例的变化(城市用地/农业用地)

Appendix 2 The variations in the proportion of land use across the areas of different slopes (urban land/farmland)

\begin{tabular}{lllll}
\hline $\begin{array}{l}\text { 坡度 } \\
\text { Slope }\end{array}$ & $\begin{array}{l}\text { 舒溪 } \\
\text { Shuxi }\end{array}$ & $\begin{array}{l}\text { 麻溪 } \\
\text { Maxi }\end{array}$ & $\begin{array}{l}\text { 浦溪 } \\
\text { Puxi }\end{array}$ & $\begin{array}{l}\text { 参考文献 } \\
\text { Reference }\end{array}$ \\
\hline$>25^{\circ}$ & $0.03 / 0.13$ & $0.13 / 0.10$ & $0.08 / 0.08$ & 陆君等, 2014 \\
$15^{\circ}-25^{\circ}$ & $0.05 / 0.22$ & $0.26 / 0.40$ & $0.37 / 0.64$ & \\
$6^{\circ}-15^{\circ}$ & $0.12 / 0.69$ & $0.60 / 1.69$ & $1.53 / 2.47$ & \\
$0^{\circ}-6^{\circ}$ & $0.43 / 2.63$ & $2.14 / 5.90$ & $7.33 / 7.94$ & \\
\hline
\end{tabular}

Lu J, Liu YF, Huang HH, Qi K, Li X, Fan ZQ (2014) Correlation analysis between land use structure and water quality of the Taiping Lake watershed in Huangshan. Journal of Fudan University (Natural Science), 53, 731-736, 746. (in Chinese with English abstract) [陆君, 刘亚风, 黄洪辉, 齐珂, 李响, 樊正球 (2014) 黄山市太平湖流域土地利用结构与河流水质相关性分析. 复旦学报(自 然科学版), 53, 731-736, 746.] 
贺佳云, 张东, 储玲, 严云志 (2021) 人为干扰对溪流鱼类功能多样性及其纵向梯度格局的影响. 生物多样性, 29, 927-937. https://www.biodiversity-science.net/CN/10.17520/biods.2020434

\section{附录3 本研究功能 $\alpha$ 和 $\beta$ 多样性指数的计算公式}

Appendix 3 The formulas of the functional $\alpha$ and $\beta$ diversity indices calculated in this study

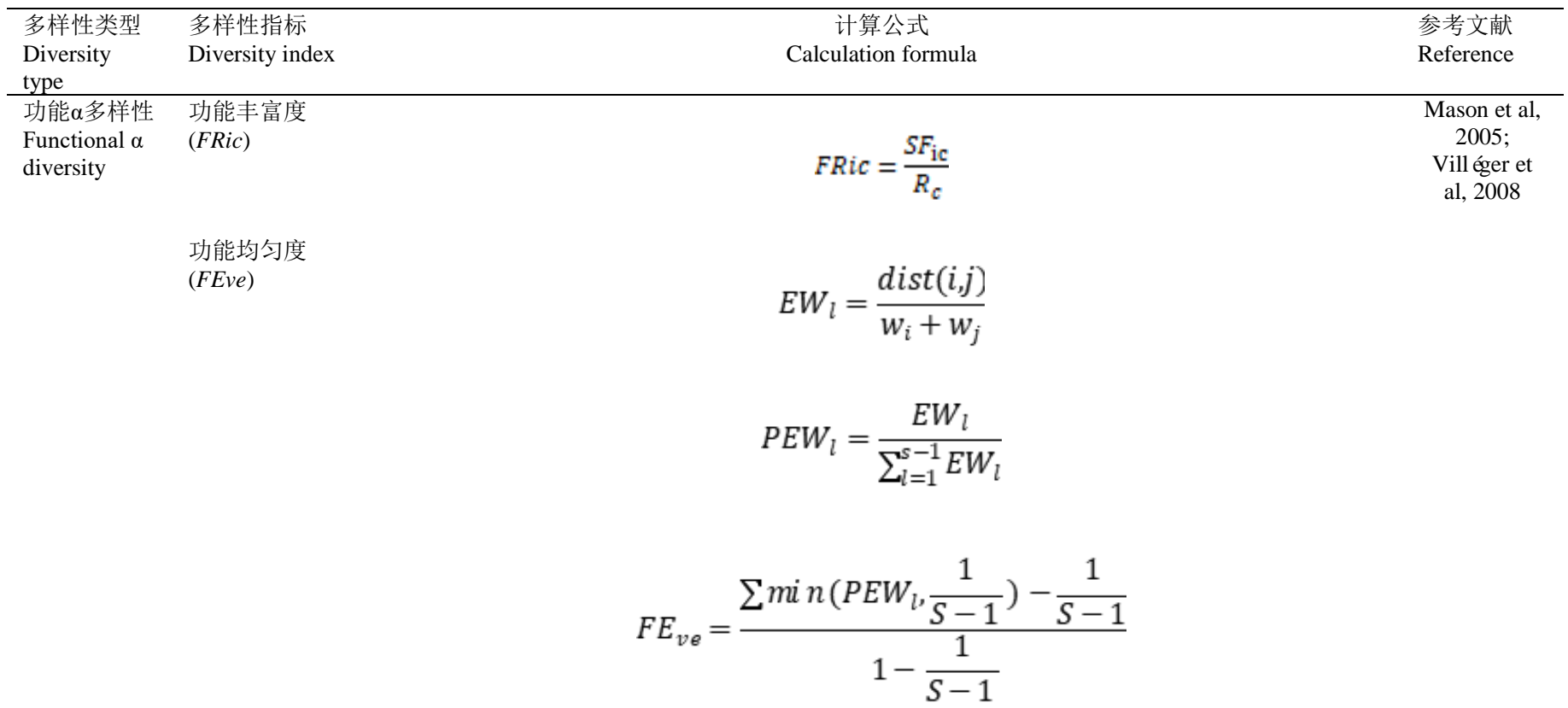

功能离散度

(FDiv)

功能 $\beta$ 多样性 $\beta \mathrm{s} ø \mathrm{r}$

\section{Functional $\beta$}

diversity

$$
\begin{gathered}
g_{k}=\frac{1}{S} \cdot \sum_{i=1}^{s} x_{i k} \\
d G_{i}=\sqrt{\sum_{k=1}^{T}\left(x_{i k}-g_{k}\right)^{2}} \\
\overline{d G}=\frac{1}{S} \sum_{i=1}^{s} d G_{i} \\
\Delta|d|=\sum_{i=1}^{s} W_{i} \times\left|d G_{i}-\overline{d G}\right| \\
F \sum_{i v}^{s}=\frac{\Delta d+\overline{d G}}{\Delta|d|+\overline{d G}}
\end{gathered}
$$

$$
\beta s ø r=\frac{\mathrm{V}(C 1)+\mathrm{V}(C 2)-2 * \mathrm{~V}(C 1 \cap C 2)}{\mathrm{V}(C 1)+\mathrm{V}(C 2)-\mathrm{V}(C 1 \cap C 2)}
$$

$\beta$ nes

$$
\beta \text { nes }=\frac{\mathrm{lV}(C 1)-\mathrm{V}(C 2) \mathrm{l}}{\mathrm{V}(C 1)+\mathrm{V}(C 2)-\mathrm{V}(C 1 \cap C 2)} * \frac{\mathrm{V}(C 1 \cap C 2)}{2 * \min (\mathrm{V}(C 1), \mathrm{V}(C 2))-\mathrm{V}(C 1 \cap C 2)} \quad \begin{gathered}
\text { Villéger et } \\
\text { al, 2013 }
\end{gathered}
$$

Btur

$$
\beta \text { tur }=\frac{2 * \min (\mathrm{V}(C 1), \mathrm{V}(C 2))-2 * \mathrm{~V}(C 1 \cap C 2)}{2 * \min (\mathrm{V}(C 1), \mathrm{V}(C 2))-\mathrm{V}(C 1 \cap C 2)}
$$


贺佳云, 张东, 储玲, 严云志 (2021) 人为干扰对溪流鱼类功能多样性及其纵向梯度格局的影响. 生物多样性, 29,927-937. https://www.biodiversity-science.net/CN/10.17520/biods.2020434

$\Delta|d|$ 为物种 $i$ 与重心的平均距离。

$C 1$ 和 $C 2$ 代表两个群落, $V(C 1)$ 和 $V(C 2)$ 分别代表这两个群落中物种功能特征所占空间体积; $V(C 1 \cap C 2)$ 代表两个群落功能空间相交部分的体 积。

$S F_{i c}$ is the niche space filled by the species within the community; $R_{c}$ is the absolute range of the character; dist $(i, j)$ is the Euclidean distance between species $i$ and $j ; S$ is species numbers; $w_{i}$ is the relative abundance of species $i ; l$ is the length of the branch; $P E W_{l}$ is weight of branch length; $x_{i k}$ is the coordinate of species $i$ on trait $k ; g_{k}$ is the center of gravity of trait $k ; d G_{i}$ is the Euclidean distance to this center of gravity; $\overline{d G}_{\text {is the mean distance of }}$ the $\mathrm{S}$ species to the center of gravity; $\Delta d$ is the sum of abundance-weighted deviances; $\Delta|d|$ is the absolute abundance-weighted deviances for distances from the center of gravity.

$C 1$ and $C 2$ represent two communities, and $\mathrm{V}(C 1)$ and $\mathrm{V}(C 2)$ is the spatial volume occupied by the functional traits of species in these two communities respectively. $\mathrm{V}(\mathrm{C} 1 \cap \mathrm{C} 2)$ is the volume of the intersection part of the functional space of two communities.

Mason NWH, Mouillot D, Lee WG, Wilson JB (2005) Functional richness, functional evenness and functional divergence: The primary components of functional diversity. Oikos, 111, 112-118.

Villéger S, Mason NWH, Mouillot D (2008) New multidimensional functional diversity indices for a multifaceted framework in functional ecology. Ecology, 89, 2290-2301.

Villéger S, Grenouillet G, Brosse S (2013) Decomposing functional $\beta$-diversity reveals that low functional $\beta$-diversity is driven by low functional turnover in European fish assemblages. Global Ecology and Biogeography, 22, 671-681. 